\title{
Integração óssea e prevalência de linhas radiotransparentes ao redor dos pinos de componentes glenoidais minimamente cimentados na artroplastia total de ombro*
}

\section{Bone Integration and Prevalence of Radiolucent Lines around the Pegs of Minimally Cemented Glenoid Components in Total Shoulder Arthroplasty}

\author{
Márcio Schiefer ${ }^{1,2}$ \\ Gláucio Siqueira ${ }^{2}$ \\ Martim Teixeira Monteiro ${ }^{2}$ \\ Geraldo Motta Filho ${ }^{2}$ \\ 1 Departamento de Ortopedia, Faculdade de Medicina, Universidade \\ Federal do Rio de Janeiro (UFRJ), Rio de Janeiro, RJ, Brasil \\ 2 Departamento de Ortopedia, Instituto Nacional de Ortopedia e \\ Traumatologia (INTO), Rio de Janeiro, RJ, Brasil \\ ${ }^{3}$ Departamento de Radiologia, Instituto Nacional de Ortopedia e \\ Traumatologia (INTO), Rio de Janeiro, RJ, Brasil
}

Alan Figueira ${ }^{2}$

Patrícia Martins Souza ${ }^{3}$

Endereço para correspondência Márcio Schiefer, MD, MSc, PhD, Rua Nóbrega, 62, Apartamento 602, Niterói, RJ, 24220-320, Brasil

(e-mail: marcioschiefer@hotmail.com).

Rev Bras Ortop 2022;57(1):120-127.

\section{Resumo}

\section{Palavras-chave}

- artroplastia de substituição

- ombro

- tomografia

- radiografia digital

- falha da prótese
Objetivos A falha do componente glenoidal é a principal causa de revisão da artroplastia total do ombro (ATO) e sua frequência parece ser influenciada pelo design do componente. $\mathrm{O}$ objetivo deste estudo foi a avaliação clínica e radiográfica (através de raios $\mathrm{X}$ e tomografia computadorizada) dos resultados da ATO com componente glenoidal minimamente cimentado.

Métodos O presente trabalho analisou ATOs realizadas com componente glenoidal Anchor Peg (DuPuy Synthes, Warsaw, IN, EUA) minimamente cimentado entre 2008 e 2013. Por um período mínimo de acompanhamento de 24 meses, escores segundo critérios da University of California at Los Angeles (UCLA) e imagens padronizadas de radiografia simples e tomografia computadorizada foram analisadas. A presença de osso entre as aletas do pino do componente central, que é um indicador de sua integração, foi avaliada nas imagens, bem como a presença de linhas radiotransparentes ao redor do componente glenoidal. Resultados Dezenove ombros de 17 pacientes foram avaliados. De acordo com o escore da UCLA, os resultados clínicos foram satisfatórios em $74 \%$ dos casos e moderados em $21 \%$ dos casos. $\mathrm{O}$ resultado foi ruim em um paciente. A integração de componentes foi

\footnotetext{
Pesquisa realizada no Instituto Nacional de Traumatologia e Ortopedia (INTO), Rio de Janeiro, RJ, Brasil.
}

recebido

17 de Fevereiro de 2020

aceito

01 de Junho de 2020

Publicado on-line

Setembro 25, 2020
DOI https://doi.org/ $10.1055 / \mathrm{s}-0040-1715509$. ISSN 0102-3616. (c) 2020. Sociedade Brasileira de Ortopedia e Traumatologia. All rights reserved.

This is an open access article published by Thieme under the terms of the Creative Commons Attribution-NonDerivative-NonCommercial-License, permitting copying and reproduction so long as the original work is given appropriate credit. Contents may not be used for commercial purposes, or adapted, remixed, transformed or built upon. (https://creativecommons.org/ licenses/by-nc-nd/4.0/)

Thieme Revinter Publicações Ltda., Rua do Matoso 170, Rio de Janeiro, RJ, CEP 20270-135, Brazil 
observada em $58 \%$ dos pacientes, sendo total em $42 \%$ e parcial em $16 \%$ dos casos. Linhas radiotransparentes foram observadas em $52 \%$ dos pacientes. Nenhuma relação entre a integração de componentes e os resultados clínicos foi detectada.

Conclusão A maioria dos pacientes submetidos à ATO com componente glenoidal minimamente cimentado apresentou resultados clínicos satisfatórios. Linhas radiotransparentes ao redor do componente glenoidal são comuns, mas não interferem nos resultados clínicos

Nível de evidência IV; Série de caso; Estudo terapêutico.

\section{Abstract}

\section{Keywords}

- arthroplasty, replacement

- shoulder

- tomography

- radiography, digital

- prosthesis failure
Objectives Glenoid component failure is the main cause of total shoulder arthroplasty (TSA) revision, and component design seems to influence the failure rate. The aim of the present study was to clinically and radiographically (through X-rays and computed tomography scan) evaluate the results of TSA using a minimally cemented glenoid component.

Methods Total shoulder arthroplasties performed using the minimally cemented Anchor Peg (DuPuy Synthes, Warsaw, IN, USA) glenoid component between 2008 and 2013 were evaluated. University of California at Los Angeles (UCLA) scores were calculated, and standardized plain film and computed tomography images were obtained, at a minimum follow-up of 24 months. The presence of bone between the fins of the central component peg, which indicates its integration, was assessed on the images, as well the presence of radiolucent lines around the glenoid component. Results Nineteen shoulders in 17 patients were available for evaluation. According to the UCLA score, clinical results were satisfactory in $74 \%$ of cases and fair in $21 \%$ of cases. One patient had a poor result. Component integration was found in $58 \%$ of patients (total in $42 \%$ and partial in $16 \%$ ). Radiolucent lines were observed in $52 \%$ of cases. No relationship was detected between component integration and clinical results.

Conclusion Satisfactory clinical results were achieved in most patients undergoing TSA using a minimally cemented glenoid component. Radiolucent lines around the glenoid component are common, but do not interfere with the clinical results.

Level of evidence IV; Case series; Treatment study.

\section{Introdução}

A artroplastia do ombro é considerada um tratamento eficaz para doenças degenerativas dessa articulação e tem resultados satisfatórios. ${ }^{1-4}$ Consequentemente, o número de artroplastias primárias do ombro aumentou de maneira significativa., Quarenta e sete mil artroplastias do ombro foram realizadas em 2008 nos Estados Unidos, o que equivale a cerca de duas vezes e meia os 19.000 procedimentos feitos em $1998 .{ }^{6}$ Ainda nos Estados Unidos, Jain e Yamaguchi ${ }^{5}$ observaram a elevação no número de artroplastias primárias do ombro de 52.397 para 67.184 entre 2009 e 2011. A indicação mais comum para o procedimento foi a osteoartrite. ${ }^{5,7}$

Resultados satisfatórios podem ser obtidos com hemiartroplastia e artroplastia total do ombro (ATO), ${ }^{8,9}$ mas a última está associada a melhores resultados em termos de saúde geral, recuperação da função, satisfação do paciente e alívio da dor. ${ }^{10,11}$ Além disso, a hemiartroplastia está associada a uma maior taxa de revisão devido à erosão glenoidal progressiva. ${ }^{10}$ Mesmo assim, a ATO não é livre de complicações e os cirurgiões devem atentar para o afrouxamento do componente glenoidal, que é a causa mais comum de reoperação após este procedimento. ${ }^{12}$ Throckmorton et al. ${ }^{2}$ não observaram diferenças clínicas ou radiológicas nos resultados da ATO com o uso de componentes glenoidais com pinos ou quilhas, mas outros estudos, em sua maioria, mostraram que a variante com pinos está associada a melhores resultados radiológicos ${ }^{10}$ e menores taxas de reoperação. ${ }^{13}$ A superfície medial do componente glenoidal também merece atenção. Implantes convexos tiveram resultados mais favoráveis do que componentes planos; além disso, há relatos que componentes metálicos aumentam o risco de complicações por afrouxamento e perda de conexão entre a base de metal e a superfície de polietileno. ${ }^{10}$

Em 2001, Wirth et al. ${ }^{14}$ usaram um modelo em cães para teste de um novo componente glenoidal de polietileno (Anchor Peg [DePuy Synthes, Warsaw, IN, EUA]) formado por pinos periféricos minimamente cimentados em torno de um longo pino central de encaixe por pressão (pressfit) com flanges radiais. Os espaços entre as flanges do pino central são preenchidos com enxerto de osso retirado da cabeça umeral removida, promovendo integração e interdigitação entre o pino e o osso nativo. ${ }^{15}$ Essa forma de fixação pode ser mais eficaz e duradoura, evitando o afrouxamento e a falha precoce da ATO. ${ }^{14}$ Estudos subsequentes obtiveram resultados encorajadores com esse tipo de componente., 15 
O objetivo deste estudo foi avaliar a integração óssea entre as faixas do pino central do componente glenoidal minimamente cimentado usando tomografia computadorizada (TC) e radiografias. Os objetivos secundários foram a identificação da distribuição das áreas radiotransparentes e sua comparação aos resultados clínicos obtidos.

\section{Materiais e métodos}

\section{Pacientes, delineamento experimental e considerações éticas}

Vinte e três pacientes foram submetidos à ATO com o componente glenoidal Anchor Peg (DePuy Synthes; Warsaw, IN, Estados Unidos) entre julho de 2008 e agosto de 2013. Este estudo intervencionista retrospectivo e transversal foi desenvolvido entre setembro de 2014 e março de 2015. Após a aprovação do comitê de ética da instituição, os 23 pacientes foram contatados e convocados para avaliação clínica e radiográfica. Seis pacientes não puderam ser contatados (um havia falecido, dois não foram encontrados e três moravam em outros estados). A amostra foi formada por 19 ombros de 17 pacientes ( 5 homens e 12 mulheres) com idade média de 65 anos (faixa etária de 42 a 80 anos).

Os critérios de inclusão foram osteoartrite primária ou secundária, realização de ATO com componente glenoidal Anchor Peg (DePuy Synthes; Warsaw, IN, Estados Unidos) e período mínimo de acompanhamento de 24 meses. Pacientes com lesões do manguito rotador e submetidos à hemiartroplastia ou que receberam outros modelos de prótese de ombro foram excluídos.

\section{Técnica cirúrgica}

Todos os pacientes foram operados sob anestesia geral e bloqueio do plexo braquial na posição em "cadeira de praia”. 0 membro superior afetado foi preparado de maneira asséptica e recoberto com campos cirúrgicos de maneira a permitir a mobilização livre durante a cirurgia. Todos os casos foram operados pela abordagem deltopeitoral. A princípio, o tendão da cabeça longa do bíceps foi identificado e a tenodese foi realizada por sutura no tendão peitoral maior. Após a liberação do tendão subescapular, a cabeça do úmero foi exposta e submetida à osteotomia com guia intramedular. Depois do preparo da diáfise umeral, a glenoide foi exposta e a capsulotomia anterior, inferior e superior foi realizada. A excisão circunferencial do lábio acetabular auxiliou a identificação dos limites exatos da borda articular. 0 centro da glenoide foi identificado para a inserção de um fio-guia e posterior confecção do orifício central ( - Fig. 1A). A cartilagem foi removida com uma serra circular, com cuidado para não causar danos ao osso subcondral ( - Fig. 1B). Em caso de necessidade de correção da versão glenoide, a fresagem excêntrica foi realizada, geralmente com a remoção de mais osso em sentido anterior do que posterior. Com o guia adequado, os três orifícios periféricos foram confeccionados ( - Fig. 1C). Após o controle apropriado do sangramento, esses orifícios foram preenchidos com cimento ósseo usando uma seringa. $O$ enxerto ósseo removido da cabeça foi inserido entre os flanges do pino central do componente glenoidal de polietileno ( - Fig. 1D), que foi, então, colocado
(-Fig. 1E). A pressão direta foi aplicada com um empurrador de glenoide para assegurar a adaptação perfeita à superfície glenoidal preparada. No lado umeral, a haste e a cabeça metálicas não cimentadas foram inseridas no canal preparado. 0 tendão subescapular foi reparado com suturas transósseas não absorvíveis da marca Orthocord (DePuy Synthes, Warsaw, IN, EUA). $O$ equilíbrio dos tecidos moles foi analisado por meio de testes de estabilidade e medida da translação anterior e posterior.

\section{Avaliação clínica}

Todos os pacientes foram submetidos à avaliação clínica, composta por anamnese e exame físico específico do ombro. Os indivíduos foram questionados sobre eventuais sintomas e a capacidade de realização de atividades diárias. 0 exame físico foi feito para análise do trofismo muscular, força e amplitudes de movimento ativo e passivo. A função do ombro foi classificada de acordo com o escore da University of California at Los Angeles (UCLA). ${ }^{16}$

\section{Avaliação radiológica}

Radiografias simples foram realizadas em incidência anteroposterior, do túnel do supraespinhoso (outlet) e axilar. Como parte do protocolo da instituição, todos os pacientes foram submetidos à TC em equipamento Brilliance 64 (Philips Medical Systems, Cleveland, OH, EUA), com aquisições em plano axial do paciente em decúbito dorsal e reconstrução com espessura de $1 \mathrm{a} 2 \mathrm{~mm}$ nos planos sagital e coronal.

Dois observadores (um radiologista com vasta experiência no campo musculoesquelético e um cirurgião de ombro e cotovelo com treinamento especializado e mais de 10 anos de experiência) avaliaram as imagens separadamente e chegaram a um consenso sobre os resultados registrados em cada caso. Nos dois tipos de imagem, a presença, localização e extensão das linhas radiotransparentes no compartimento glenoidal foram avaliadas como sugerido por Vidil et al. ${ }^{3}$ Os espaços entre os flanges do pino central foram examinados para determinação da presença de trabéculas ósseas sugestivas de crescimento tecidual. O implante foi considerado totalmente integrado ao apresentar trabéculas ósseas em todos os espaços (-Fig. 2), parcialmente integrado quando o crescimento era observado em apenas alguns espaços ( - Fig. 3) e não integrado em caso de ausência de trabéculas ( - Fig. 4 ).

\section{Análise estatística}

Os dados foram armazenados e analisados com o programa SPSS Statistics for Window Version 20.0. (IBM Corp., Armonk, NY, EUA). Uma extensão de Freeman-Halton do teste exato de Fisher avaliou a distribuição de linhas radiotransparentes entre as diferentes áreas do componente glenoidal. O teste de Spearman identificou correlações entre a presença dessas linhas nos exames radiológicos e os resultados clínicos, determinados principalmente pelo escore de ombro da UCLA.

\section{Resultados}

A - Tabela 1 mostra os dados obtidos neste estudo. A integração óssea do pino central foi observada em 58\% dos 

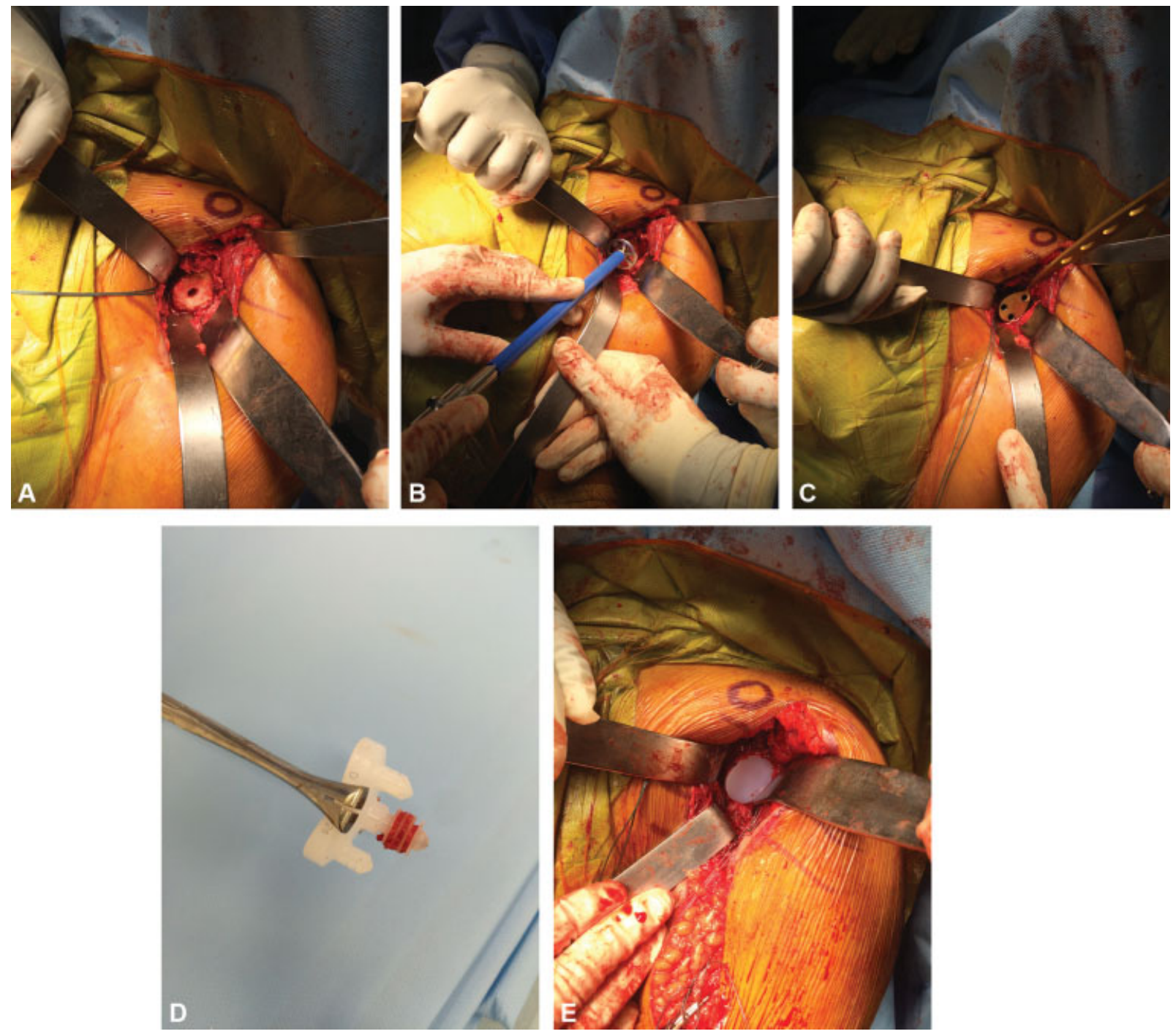

Fig. 1 Abordagem deltopeitoral do ombro esquerdo, mostrando (A) orifício central da glenoide; (B) preparo da glenoide; (C) posicionamento do guia de perfuração para confecção dos orifícios periféricos; (D) enxerto ósseo autólogo entre as aletas do pino central; (E) aspecto final do componente glenoidal implantado.
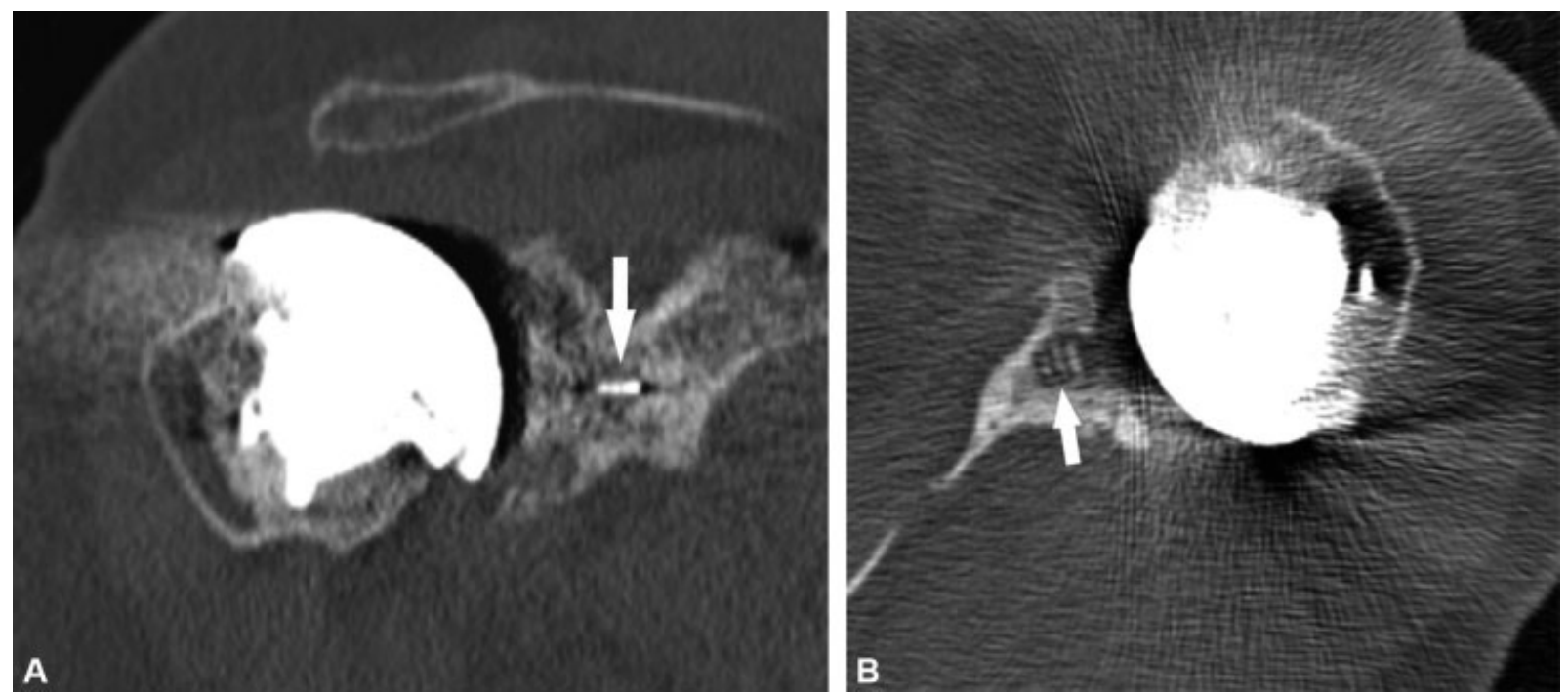

Fig. 2 Imagens de tomografia computadorizada mostrando a integração óssea completa do pino central. Observe a presença de osso em todos os espaços entre as aletas do pino central (setas) em plano coronal (A) e axial (B). 


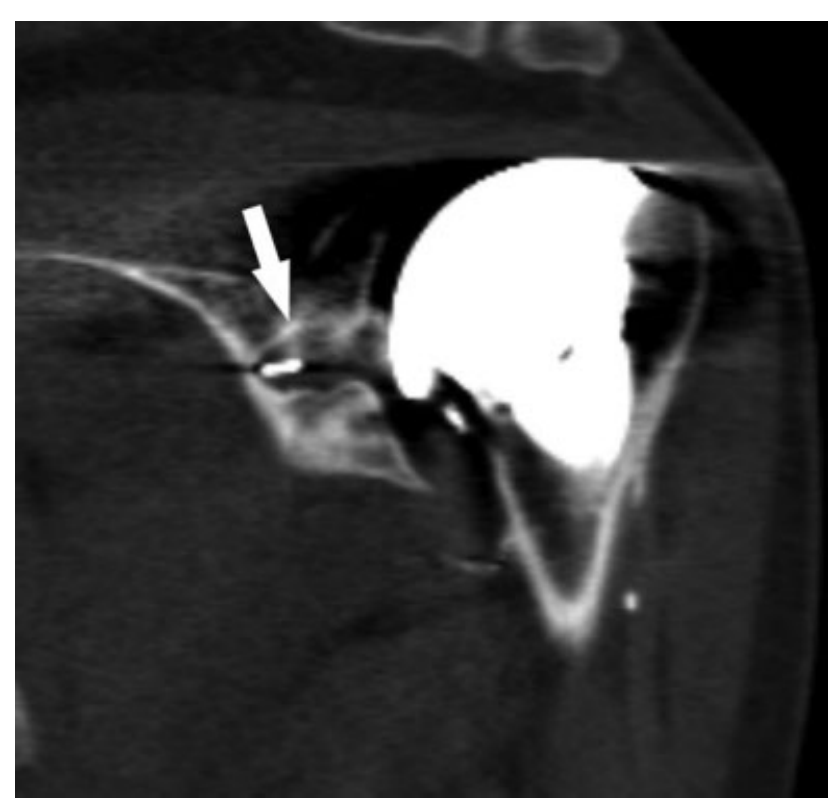

Fig. 3 Imagem coronal de tomografia computadorizada do ombro esquerdo mostrando integração óssea parcial do pino central. Observe a presença de osso em alguns espaços entre as aletas do pino central (seta).

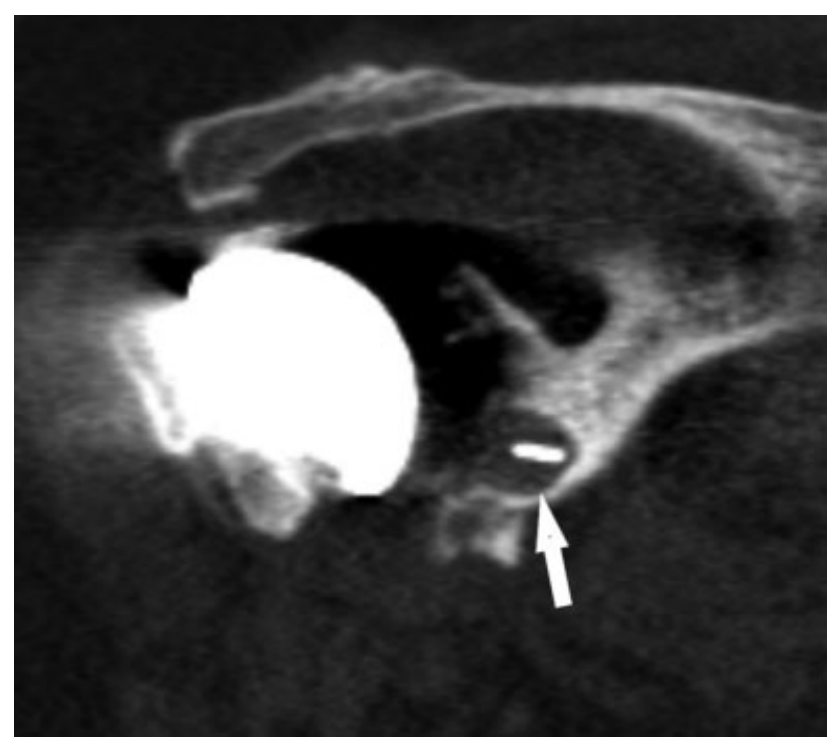

Fig. 4 Imagem coronal de tomografia computadorizada de ombro direito mostrando ausência de integração óssea do pino central. Não há osso entre as aletas do pino central (seta).

casos, sendo parcial em $16 \%$ e total em $42 \%$ dos pacientes. Os demais $42 \%$ de casos não apresentaram integração óssea.

Linhas radiotransparentes foram observadas em 10 (52\%) casos, nas áreas 4, 5 e 8 (-Tabela 1 ). A área 5, ao redor do pino central, foi o local mais comum de linhas radiotransparentes $(p=0,008)$. As linhas tinham mais de $1,5 \mathrm{~mm}$ em apenas um caso. Essas linhas foram mais comuns em casos sem integração óssea do pino central, mas sem diferença significativa.

Setenta e nove por cento dos casos foram operados por osteoartrite primária e $21 \%$ foram submetidos à cirurgia por osteoartrite secundária; todos os casos eram sequelas de trauma. Três pacientes apresentaram fraturas fechadas e um tinha lesão causada por arma de fogo. Não houve associação significativa entre a etiologia da fratura e a presença de linhas radiotransparentes, embora estas linhas não tenham sido observadas nos quatro casos de osteoartrite secundária.

De acordo com a escala funcional da UCLA, ${ }^{16}$ os resultados foram satisfatórios em 73,69\% dos casos, regulares em $21 \%$ e maus em 1 paciente (5,3\%) (-Tabela 2 ). Não houve correlação entre a idade, a duração dos sintomas antes da cirurgia ou a presença de linhas de integração óssea ou radiotransparentes e os resultados clínicos de acordo com a escala da UCLA (-Tabela 3). Durante o período de acompanhamento, nenhum paciente apresentou complicação relacionada ou não à cirurgia.

\section{Discussão}

Embora as taxas de sobrevida do componente umeral sejam elevadas, ${ }^{1}$ problemas com a glenoide, muitos de difícil resolução, ainda são a principal causa de revisão da ATO. ${ }^{10,17}$ Bonnevialle et al. ${ }^{18}$ demonstraram uma alta frequência de recidiva do afrouxamento após a revisão da ATO. Em busca de um design de maior durabilidade, Wirth et al. ${ }^{14}$ testaram um novo componente glenoidal com um pino central e três pinos periféricos em cães. Somente os pinos periféricos são cimentados; o pino central é inserido sob pressão, na tentativa de evitar os efeitos deletérios do cimento no estoque ósseo glenoidal ${ }^{19}$ e aumentar a fixação ao osso nativo, já que os espaços entre os flanges do pino central permitem o crescimento ósseo.

Poucos estudos analisaram a integração óssea do componente glenoidal Anchor Peg em pacientes submetidos à ATO. Embora cerca de 33.000 desses componentes tenham sido implantados em todo o mundo desde $2011,{ }^{15}$ poucos artigos relataram os resultados obtidos. Isso corrobora o fato de que apenas $5 \%$ de todas as artroplastias do ombro são objeto de publicação científica. $^{7}$

Os resultados radiográficos descritos em estudos do componente glenoidal com pino central e aletas não cimentadas e de componentes com outros designs são heterogêneos com linhas radiotransparentes relatadas em 0 a $94 \%$ dos casos. ${ }^{20} \mathrm{Em}$ um estudo radiológico simples de pacientes que receberam componentes Anchor Peg, Churchill et al. ${ }^{21}$ relataram integração óssea em $85 \%$ dos casos e uma incidência de linhas radiotransparentes de $25 \%$. Por sua vez, Groh, ${ }^{22}$ em outro estudo radiológico simples, observou integração óssea em 24 de 83 (29\%) pacientes. Arnold et al. ${ }^{23}$ avaliaram imagens de TC, como neste estudo, e relataram integração óssea em 23 de 35 (65\%) pacientes e linhas radiotransparentes em 11 (31\%) casos. Wirth et al. ${ }^{15}$ analisaram a presença de linhas radiotransparentes ao redor do componente glenoidal em radiografias simples e relataram uma incidência de $68 \%$ (30 de 44 casos). Vidil et al. ${ }^{3}$ observaram integração óssea em 21 de 26 (80\%) casos operados e avaliados por TC. Wijeratna et al. ${ }^{24}$ relataram integração óssea em 68 dos 83 ombros que receberam o componente glenoidal Anchor Peg e 78 pacientes não apresentaram linhas radiotransparentes à TC. Kilian et al. ${ }^{17} \mathrm{e}$ Merolla et al. ${ }^{25}$ observaram taxas menores de crescimento ósseo, de 25\% e 23\%, respectivamente. Nesse último trabalho, linhas radiotransparentes progressivas foram detectadas em 
Tabela 1 Resultados gerais

\begin{tabular}{|c|c|c|c|c|c|c|c|c|c|}
\hline Paciente & Sexo & $\begin{array}{l}\text { Idade } \\
\text { (anos) }\end{array}$ & $\begin{array}{l}\text { Membro } \\
\text { dominante }\end{array}$ & $\begin{array}{l}\text { Etiologia } \\
\text { da AO }\end{array}$ & $\begin{array}{l}\text { Acompanhamento } \\
\text { (meses) }\end{array}$ & 10 & LR & $\begin{array}{l}\text { Zona de } \\
\text { Vidil de LR }\end{array}$ & $\begin{array}{l}\text { Escore } \\
\text { UCLA }\end{array}$ \\
\hline 1 & $M$ & 73 & Não & Primária & 57 & Ausente & Sim & 5 & 34 \\
\hline 2 & $\mathrm{~F}$ & 75 & Não & Primária & 46 & Completa & Não & 0 & 29 \\
\hline 3 & $\mathrm{~F}$ & 78 & Não & Primária & 13 & Completa & Sim & 5 & 32 \\
\hline 4 & $\mathrm{~F}$ & 76 & Sim & Primária & 45 & Parcial & Sim & 5 & 32 \\
\hline 5 & $\mathrm{~F}$ & 80 & Sim & Primária & 14 & Completa & Não & 0 & 22 \\
\hline 6 & $\mathrm{M}$ & 42 & Sim & Primária & 16 & Parcial & Sim & 5 & 25 \\
\hline 7 & $\mathrm{~F}$ & 59 & Sim & Primária & 12 & Ausente & Não & 0 & 33 \\
\hline 8 & $\mathrm{~F}$ & 59 & Não & Primária & 48 & Completa & Não & 0 & 34 \\
\hline 9 & $\mathrm{~F}$ & 58 & Não & Secundária & 27 & Completa & Não & 0 & 25 \\
\hline 10 & $\mathrm{~F}$ & 64 & Não & Primária & 55 & Ausente & Não & 0 & 28 \\
\hline 11 & $\mathrm{~F}$ & 73 & Sim & Primária & 68 & Ausente & Sim & 8 & 28 \\
\hline 12 & $\mathrm{~F}$ & 70 & Sim & Primária & 27 & Completa & Não & 0 & 30 \\
\hline 13 & $\mathrm{~F}$ & 70 & Não & Primária & 48 & Ausente & Sim & 5 & 31 \\
\hline 14 & $\mathrm{~F}$ & 59 & Sim & Primária & 53 & Ausente & Sim & 5 & 32 \\
\hline 15 & $\mathrm{M}$ & 51 & Não & Secundária & 55 & Completa & Sim & 4 & 17 \\
\hline 16 & $\mathrm{~F}$ & 77 & Sim & Primária & 71 & Ausente & Sim & 5 & 32 \\
\hline 17 & $\mathrm{M}$ & 60 & Não & Secundária & 35 & Parcial & Não & 0 & 32 \\
\hline 18 & $\mathrm{~F}$ & 67 & Sim & Secundária & 17 & Completa & Não & 0 & 28 \\
\hline 19 & $\mathrm{M}$ & 53 & Sim & Primária & 19 & Ausente & Sim & 5 & 27 \\
\hline
\end{tabular}

Abreviaturas: F, feminino; M, masculino; OA, osteoartrite; IO, integração óssea; LR, linha radiotransparente; UCLA, University of California at Los Angeles.

Tabela 2 Distribuição dos escores da UCLA

\begin{tabular}{|l|l|}
\hline Escore UCLA & Número de pacientes (\%) \\
\hline Excelente $(34-35)$ & $2(10,53)$ \\
\hline Bom $(28-33)$ & $12(63,16)$ \\
\hline Moderado $(21-27)$ & $4(21,05)$ \\
\hline Baixo $(<20)$ & $1(5,26)$ \\
\hline
\end{tabular}

UCLA, University of California at Los Angeles.

Tabela 3 Correlações entre a idade do paciente, a duração do acompanhamento e o escore da UCLA $(n=19)$

\begin{tabular}{|l|l|l|l|}
\hline & & $\begin{array}{l}\text { Duração do } \\
\text { acompanhamento }\end{array}$ & $\begin{array}{l}\text { Escore } \\
\text { UCLA }\end{array}$ \\
\hline \multirow{2}{*}{ Idade } & $r$ & 0,093 & 0,266 \\
\cline { 2 - 4 } & $p$ & 0,704 & 0,272 \\
\hline \multirow{2}{*}{$\begin{array}{l}\text { Duração do } \\
\text { acompanhamento }\end{array}$} & $r$ & & 0,163 \\
\cline { 2 - 4 } & $p$ & & 0,506 \\
\hline
\end{tabular}

UCLA, University of California at Los Angeles.

28 de 30 componentes glenoidais. Em nosso estudo, a integração óssea foi observada em 11 de 19 (58\%) pacientes, uma taxa média em relação aos estudos anteriores.

Acreditamos que a variação na incidência relatada de linhas radiotransparentes se deva a vários fatores. Alguns estudos avaliaram apenas radiografias, ${ }^{15,21,22}$ enquanto outros usaram
TC. ${ }^{3,23}$ Mesmo estudos que empregaram o mesmo método radiológico podem ter utilizado diferentes protocolos de avaliação. A suscetibilidade dos estudos de imagem do componente glenoidal a erros, especialmente em caso de análise de radiografias simples, está bem documentada; a interpretação por TCé mais precisa. ${ }^{24,26,27}$ Outro fator importante é a duração do acompanhamento, pois a prevalência de linhas radiotransparentes aumenta com o tempo. ${ }^{15}$ Por fim, um único cirurgião experiente realizou todos os procedimentos em alguns estudos, mas não em todos. Segundo Lazarus et al., ${ }^{20}$ os resultados radiológicos do implante de componente glenoidal foram melhores quando o procedimento foi realizado pelo cirurgião mais experiente; esse fator pode contribuir para a discrepância entre os relatos. A influência da técnica de cimentação na incidência de linhas radiotransparentes ao redor do componente glenoidal foi descrita. ${ }^{28,29}$ Assim, a aplicação de uma melhor técnica de cimentação, geralmente por um cirurgião mais experiente, poderia impedir o aparecimento desses sinais radiotransparentes. Esse fator pode explicar parcialmente a ausência de linhas radiotransparentes nos pacientes com osteoartrite secundária deste estudo, embora esses casos sejam tecnicamente mais exigentes e o bom posicionamento dos componentes glenoidais seja mais difícil do que em indivíduos com osteoartrite primária. Acreditamos que a realização desses procedimentos pelos cirurgiões mais experientes de nossa instituição também explique os resultados.

A implicação exata da presença de linhas radiotransparentes ao redor do componente glenoidal não é conhecida. Essas 
linhas são observadas em até $94 \%$ dos $\operatorname{casos}^{20}$ e sua presença não parece influenciar os resultados clínicos. ${ }^{1,21,24,25}$ Aqui, não observamos uma influência dessa variável no escore da UCLA. Além disso, a definição de afrouxamento de componentes é bastante variável entre os estudos. ${ }^{1,20,30}$ A maioria dos autores considerou a espessura de uma linha radiotransparente e sua extensão por diferentes regiões do componente. No presente estudo, nenhum paciente atendeu aos critérios de afrouxamento do componente. Apenas um paciente apresentou uma linha radiotransparente maior que $1,5 \mathrm{~mm}$, localizada exclusivamente ao redor do pino central. Esse mesmo paciente apresentou bom resultado clínico de acordo com o escore da UCLA. Nosso estudo revelou fraca correlação entre os achados clínicos e radiográficos, embora um período maior de acompanhamento possa ser necessário. Idade, duração dos sintomas e presença de integração óssea e linhas radiotransparentes não influenciaram os resultados clínicos. Achados semelhantes foram relatados por Wijeratna et al. ${ }^{24}$ No entanto, um estudo recente mostrou que pacientes cujos componentes glenoidais apresentavam linhas radiotransparentes demonstraram menor melhora na elevação para frente e em todos os resultados relatados pelos pacientes ${ }^{31}$

Embora a ausência de integração óssea não tenha consequências clínicas comprovadas, ${ }^{24}$ sua presença é considerada desejável em todos os pacientes. Em uma análise radioestereométrica, Nuttal et al. ${ }^{32}$ avaliaram o movimento do componente glenoidal Anchor Peg em 11 pacientes submetidos à ATO. Os pacientes foram divididos em dois grupos de acordo com os resultados: um grupo apresentou movimentação inicial dos componentes e ausência de integração óssea ao acompanhamento de 12 meses e o outro grupo não demonstrou movimento e evoluiu com integração óssea. Merolla et al. ${ }^{25}$ mostraram que pacientes com crescimento ósseo em direção ao pino central eram menos propensos a apresentar linhas radiotransparentes. Porém, como também ocorreu aqui, Churchill et al. ${ }^{21}$ e Arnold et al. ${ }^{23}$ não encontraram relação entre integração óssea e resultados clínicos determinados pela escala de Constant e pelo teste simples do ombro.

Embora este estudo tenha pontos fortes, ele também possui várias limitações. Primeiro, nossa amostra era pequena, em parte devido à seleção rigorosa e cuidadosa dos pacientes submetidos à ATO em nossa instituição e pela exclusão de procedimentos realizados com outros tipos de componente glenoidal. A amostra também era pequena devido à tendência mundial de redução da proporção entre artroplastias anatômicas e reversas do ombro, ${ }^{5}$ refletida de forma significativa em nossa instituição. Outra limitação é a não disponibilização imediata de radiografias e imagens de TC do período pós-operatório de todos os pacientes; isso impediu a identificação de todos os indivíduos com linhas radiotransparentes imediatamente após a cirurgia, o que indicaria a inadequação do componente glenoidal.

Até onde temos conhecimento, o presente estudo é o primeiro conduzido em nosso país a comparar resultados clínicos e radiográficos de pacientes submetidos à ATO com o componente glenoidal Anchor Peg. O uso de imagens de TC permitiu a análise mais precisa da integração óssea no pino central e a identificação de linhas radiotransparentes. Outra vantagem foi a realização de cirurgias por profissionais com diferentes níveis de especialização. Acreditamos que esse fator favorece a reprodutibilidade, diferentemente dos resultados de outras séries, em que o cirurgião mais experiente do grupo operou todos os pacientes. ${ }^{15,23}$ Além disso, o mesmo pesquisador avaliou todos os pacientes, reduzindo o viés de mensuração. Por fim, a análise consensual das imagens por dois profissionais, sendo um radiologista com vasta experiência no campo musculoesquelético, foi um ponto forte do estudo. Concordamos com Arnold et al. ${ }^{23}$ que a avaliação de concordância inter e intraobservador não é necessária, devido às excelentes taxas de concordância demonstradas por Yian et al., ${ }^{33}$ principalmente com o uso da TC.

\section{Conclusão}

Embora a integração óssea do pino central tenha sido observada em pouco mais de metade dos casos, a maioria dos pacientes submetidos à ATO com componente glenoidal minimamente cimentado apresentou resultados clínicos satisfatórios. Linhas radiotransparentes ao redor do componente glenoidal foram comumente observadas, mas não correlacionadas aos resultados clínicos.

Conflito de interesses

Os autores declaram não haver conflito de interesses.

\section{Referências}

1 Raiss P, Schmitt M, Bruckner T, et al. Results of cemented total shoulder replacement with a minimum follow-up of ten years. J Bone Joint Surg Am 2012;94(23):e1711-e10

2 Throckmorton TW, Zarkadas PC, Sperling JW, Cofield RH. Pegged versus keeled glenoid components in total shoulder arthroplasty. J Shoulder Elbow Surg 2010;19(05):726-733

3 Vidil A, Valenti P, Guichoux F, Barthas JH. CT scan evaluation of glenoid component fixation: a prospective study of 27 minimally cemented shoulder arthroplasties. Eur J Orthop Surg Traumatol 2013;23(05):521-525

4 De Wilde L, Dayerizadeh N, De Neve F, Basamania C, Van Tongel A. Fully uncemented glenoid component in total shoulder arthroplasty. J Shoulder Elbow Surg 2013;22(10):e1-e7

5 Jain NB, Yamaguchi K. The contribution of reverse shoulder arthroplasty to utilization of primary shoulder arthroplasty. J Shoulder Elbow Surg 2014;23(12):1905-1912

6 Kim SH, Wise BL, Zhang Y, Szabo RM. Increasing incidence of shoulder arthroplasty in the United States. J Bone Joint Surg Am 2011;93(24):2249-2254

7 Motta Filho G, Galvão MV, Monteiro M, Cohen M, Brandão B. Registro de artroplastias do ombro. Rev Bras Ortop 2009;44(02):125-133

8 Gadea F, Alami G, Pape G, Boileau P, Favard L. Shoulder hemiarthroplasty: outcomes and long-term survival analysis according to etiology. Orthop Traumatol Surg Res 2012;98(06):659-665

9 Sperling JW, Cofield RH, Rowland CM. Minimum fifteen-year follow-up of Neer hemiarthroplasty and total shoulder arthroplasty in patients aged fifty years or younger. J Shoulder Elbow Surg 2004;13(06):604-613

10 Boileau P, Sinnerton RJ, Chuinard C, Walch G. Arthroplasty of the shoulder. J Bone Joint Surg Br 2006;88(05):562-575

11 Bryant D, Litchfield R, Sandow M, Gartsman GM, Guyatt G, Kirkley A. A comparison of pain, strength, range of motion, and functional outcomes after hemiarthroplasty and total shoulder arthroplasty in patients with osteoarthritis of the shoulder. A systematic review and meta-analysis. J Bone Joint Surg Am 2005;87(09):1947-1956 
12 Matsen FA 3rd, Clinton J, Lynch J, Bertelsen A, Richardson ML. Glenoid component failure in total shoulder arthroplasty. J Bone Joint Surg Am 2008;90(04):885-896

13 Vavken P, Sadoghi P, von Keudell A, Rosso C, Valderrabano V, Müller AM. Rates of radiolucency and loosening after total shoulder arthroplasty with pegged or keeled glenoid components. J Bone Joint Surg Am 2013;95(03):215-221

14 Wirth MA, Korvick DL, Basamania CJ, Toro F, Aufdemorte TB, Rockwood CA Jr. Radiologic, mechanical, and histologic evaluation of 2 glenoid prosthesis designs in a canine model. J Shoulder Elbow Surg 2001;10(02):140-148

15 Wirth MA, Loredo R, Garcia G, Rockwood CA Jr, Southworth C, Iannotti JP. Total shoulder arthroplasty with an all-polyethylene pegged bone-ingrowth glenoid component: a clinical and radiographic outcome study. J Bone Joint Surg Am 2012;94(03):260-267

16 Amstutz HC, Sew Hoy AL, Clarke IC. UCLA anatomic total shoulder arthroplasty. Clin Orthop Relat Res 1981;(155):7-20

17 Kilian CM, Morris BJ, Sochacki KR, et al. Radiographic comparison of finned, cementless central pegged glenoid component and conventional cemented pegged glenoid component in total shoulder arthroplasty: a prospective randomized study. J Shoulder Elbow Surg 2018;27(6S):S10-S16

18 Bonnevialle N, Melis B, Neyton L, et al. Aseptic glenoid loosening or failure in total shoulder arthroplasty: revision with glenoid reimplantation. J Shoulder Elbow Surg 2013;22(06):745-751

19 Churchill RS, Boorman RS, Fehringer EV, Matsen FA III. Glenoid cementing may generate sufficient heat to endanger the surrounding bone. Clin Orthop Relat Res 2004;(419):76-79

20 Lazarus MD, Jensen KL, Southworth C, Matsen FA III. The radiographic evaluation of keeled and pegged glenoid component insertion. J Bone Joint Surg Am 2002;84(07):1174-1182

21 Churchill RS, Zellmer C, Zimmers HJ, Ruggero R. Clinical and radiographic analysis of a partially cemented glenoid implant: five-year minimum follow-up. J Shoulder Elbow Surg 2010;19(07):1091-1097

22 Groh GI. Survival and radiographic analysis of a glenoid component with a cementless fluted central peg. J Shoulder Elbow Surg 2010;19(08):1265-1268

23 Arnold RM, High RR, Grosshans KT, Walker CW, Fehringer EV. Bone presence between the central peg's radial fins of a partially cemented pegged all poly glenoid component suggest few radiolucencies. J Shoulder Elbow Surg 2011;20(02):315-321

24 Wijeratna M, Taylor DM, Lee S, Hoy G, Evans MC. Clinical and radiographic results of an all-polyethylene pegged boneingrowth glenoid component. J Bone Joint Surg Am 2016;98 (13):1090-1096

25 Merolla G, Ciaramella G, Fabbri E, Walch G, Paladini P, Porcellini G. Total shoulder replacement using a bone ingrowth central peg polyethylene glenoid component: a prospective clinical and computed tomography study with short- to mid-term followup. Int Orthop 2016;40(11):2355-2363

26 Gregory T, Hansen U, Khanna M, et al. A CT scan protocol for the detection of radiographic loosening of the glenoid component after total shoulder arthroplasty. Acta Orthop 2014;85(01):91-96

27 Hoenecke HR Jr, Hermida JC, Flores-Hernandez C, D'Lima DD. Accuracy of CT-based measurements of glenoid version for total shoulder arthroplasty.J Shoulder Elbow Surg 2010;19(02):166-171

28 Barwood S, Setter KJ, Blaine TA, Bigliani LU. The incidence of early radiolucencies about a pegged glenoid component using cement pressurization. J Shoulder Elbow Surg 2008;17(05):703-708

29 Choi T, Horodyski M, Struk AM, Sahajpal DT, Wright TW. Incidence of early radiolucent lines after glenoid component insertion for total shoulder arthroplasty: a radiographic study comparing pressurized and unpressurized cementing techniques. J Shoulder Elbow Surg 2013;22(03):403-408

30 Sperling JW, Cofield RH, O'Driscoll SW, Torchia ME, Rowland CM. Radiographic assessment of ingrowth total shoulder arthroplasty. J Shoulder Elbow Surg 2000;9(06):507-513

31 Schoch BS, Wright TW, Zuckerman JD, et al. Glenoid component lucencies are associated with poorer patient-reported outcomes following anatomic shoulder arthroplasty. J Shoulder Elbow Surg 2019;28(10):1956-1963

32 Nuttall D, Haines JF, Trail IA. The early migration of a partially cemented fluted pegged glenoid component using radiostereometric analysis. J Shoulder Elbow Surg 2012;21(09):1191-1196

33 Yian EH, Werner CML, Nyffeler RW, et al. Radiographic and computed tomography analysis of cemented pegged polyethylene glenoid components in total shoulder replacement. J Bone Joint Surg Am 2005;87(09):1928-1936 